\title{
Pathophysiological roles of adrenomedullin-RAMP2 system in acute and chronic cerebral ischemia
}

\author{
Kyoko Igarashi ${ }^{a}$, Takayuki Sakurai ${ }^{a}$, Akiko Kamiyoshi ${ }^{a}$, Yuka Ichikawa-Shindo ${ }^{a}$, \\ Hisaka Kawate $^{a}$, Akihiro Yamauchi ${ }^{a}$, Yuichi Toriyama ${ }^{a}$, Megumu Tanaka ${ }^{a}$, Tian Liu ${ }^{a}$, \\ Xian Xian ${ }^{a}$, Akira Imai ${ }^{a}$, Liuyu Zhai ${ }^{a}$, Shinji Owa ${ }^{a}$, Teruhide Koyama ${ }^{a}$, Ryuichi Uetake ${ }^{a}$, \\ Masafumi Ihara $^{\mathrm{b}}$, Takayuki Shindo ${ }^{\mathrm{a}, *}$ \\ a Department of Cardiovascular Research, Shinshu University Graduate School of Medicine, Matsumoto, Japan \\ ${ }^{\mathrm{b}}$ Department of Regenerative Medicine, Research Institute of Biomedical Research and Innovation, Kobe, Japan
}

\section{A R T I C L E I N F O}

Article history:

Received 26 June 2014

Received in revised form 25 August 2014

Accepted 25 August 2014

Available online 22 September 2014

\section{Keywords:}

Adrenomedullin

Receptor activity-modifying protein

Cerebral ischemia

Blood vessel

\begin{abstract}
A B S T R A C T
The accessory protein RAMP2 is a component of the CLR/RAMP2 dimeric adrenomedullin (AM) receptor and is the primary determinant of the vascular functionality of AM. RAMP2 is highly expressed in the brain; however, its function there remains unclear. We therefore used heterozygous RAMP2 knockout (RAMP2+/-) mice, in which RAMP2 expression was reduced by half, to examine the actions of the endogenous AM-RAMP2 system in cerebral ischemia. To induce acute or chronic ischemia, mice were subjected to middle cerebral artery occlusion (MCAO) or bilateral common carotid artery stenosis (BCAS), respectively. In RAMP2+/ - mice subjected to MCAO, recovery of cerebral blood flow (CBF) was slower than in WT mice. AM gene expression was upregulated after infarction in both genotypes, but the increase was greater in RAMP2 $+\mid-$ mice. Pathological analysis revealed severe nerve cell death and demyelination, and a higher level of oxidative stress in RAMP2 $+/-$ mice. In RAMP2 $+/-$ mice subjected to BCAS, recovery of cerebral perfusion was slower and less complete than in WT mice. In an 8-arm radial maze test, RAMP2+/- mice required more time to solve the maze and showed poorer reference memory. They also showed greater reductions in nerve cells and less compensatory capillary growth than WT mice. These results indicate the AM-RAMP2 system works to protect nerve cells from both acute and chronic cerebral ischemia by maintaining CBF, suppressing oxidative stress, and in the case of chronic ischemia, enhancing capillary growth.
\end{abstract}

() 2014 Elsevier Inc. All rights reserved.

\section{Introduction}

Although the mortality rates for cerebrovascular diseases have declined in recent years, thanks to advances in treatment, they continue to be important life-threatening ailments. Furthermore, the prevention and management of the aftereffects and disability caused by cerebrovascular diseases are urgent social problems,

Abbreviations: AM, adrenomedullin; RAMP, receptor activity-modifying protein; CLR, calcitonin-receptor-like receptor; CNS, central nervous system; WT, wild-type; MCAO, middle cerebral artery occlusion; BCAS, bilateral carotid artery stenosis; CBF, cerebral blood flow; 8-OHdG, 8-hydroxy-2'-deoxyguanosine; GFAP, glial fibrillary acidic protein.

* Corresponding author at: Department of Cardiovascular Research, Shinshu University Graduate School of Medicine, Asahi 3-1-1, Matsumoto, Nagano 390-8621, Japan. Tel.: +81263 37 2578; fax: +81263373437.

E-mail address: tshindo@shinshu-u.ac.jp (T. Shindo). especially in aging societies. Cerebrovascular diseases include cerebral infarction, cerebral hemorrhage, subarachnoid hemorrhage, subdural hematoma, and moyamoya disease, among others, with cerebral infarction affecting the largest number of patients. In cerebral infarction, a thrombus is formed in a brain blood vessel and the neurons supplied by that vessel undergo ischemic damage, leading to motility and sensory dysfunction. In addition, cerebral infarctions can also be the cause of vascular dementia resulting from chronic cerebral hypoperfusion. Next to Alzheimer's disease, vascular dementia is the second most common form of dementia; however, no effective treatments for vascular dementia have yet been established. It appears that improving the prognosis of patients with cerebrovascular disease will require new approaches to treatment, perhaps focusing on vascular homeostasis.

Adrenomedullin (AM) was originally identified as a vasodilatory peptide from human pheochromocytoma [11]. It is now known that AM is expressed and secreted by various organs and exhibits a 
Table 1

Primers and probes used for quantitative real-time RT-PCR

\begin{tabular}{ll}
\hline AM Forward & CTACCGCCAGAGCATGAACC \\
AM Reverse & GAAATGTGCAGGTCCGAA \\
AM Probe & CCCGCAGCAATGGATGCCG \\
CLR Forward & AGGCGTTACCTGCACACACT \\
CLR Reverse & CAGGAAGCAGAGGAAACCCC \\
CLR Probe & ATCGTGGTGGCTGTGTTTGCGGAG \\
RAMP2 Forward & GCAGCCCACCTTCTCTGATC \\
RAMP2 Reverse & AACGGGATGAGGCAGATGG \\
RAMP2 Probe & CCCAGAGGATGTGCTCCTGGCCAT \\
RAMP3 Forward & TGCAACGAGACAGGGATGC \\
RAMP3 Reverse & GCATCATGTCAGCGAAGGC \\
RAMP3 Probe & AGAGGCTGCCTCGCTGTGGGAA \\
IL-6 Forward & CCCAATTCCAATGCTCTCC \\
IL-6 Reverse & TGAATTGGATGGTCTTGGTCC \\
IL-1 $\beta$ Forward & CTACAGGCTCCGAGATGAACAAC \\
IL-1 $\beta$ Reverse & TCCATTGAGGTGGAGAGCTTTC \\
TNF- $\alpha$ Forward & ACGGCATGGATCTCAAAGAC \\
TNF- $\alpha$ Reverse & AGATAGCAAATCGGCTGACG \\
\hline
\end{tabular}

wide array of bioactivities. For example, AM suppresses antioxidative stress [30,31], inflammation [8] and apoptosis [23,37], whereas it stimulates the proliferation, migration and differentiation of various cell types $[9,10,17]$. It is also involved in the regulation of hormone secretion $[24,25]$ and exerts organ-protective effects $[21,22]$, as well as its vasodilatory effect. We previously reported that homozygous AM knockout ( $\mathrm{AM}-/-$ ) mice die in utero due to defective vascular development, which highlights the angiogenic function of AM [32].

AM is also distributed in the central nervous system (CNS); cerebrovascular cells, neurons and glial cells all secrete AM. Furthermore, intracerebroventricular administration of AM leads to reductions in drinking, salt intake and eating behavior [18,26,34]. Recently, Maki et al. reported that tissue injury after chronic cerebral ischemia was reduced and cognitive function was improved in AM-overexpressing mice as a result of enhanced neovascularization $[13,14]$. Although these results sparked much interest in the potential clinical application of AM for the treatment of cerebrovascular diseases, the very short half-life of AM in blood limits its applicability for chronic diseases, which require long-term therapy. We have therefore been concentrating on the AM receptor system as an alternative therapeutic target. The AM receptor is a heterodimer composed of calcitonin receptor-like receptor (CLR; a seven-transmembrane G-protein coupled receptor) and an accessory protein called receptor activity-modifying protein (RAMP). Three RAMP isoforms have been identified (RAMP1, 2, 3). We previously showed that homozygous RAMP2 knockout (RAMP2-/-) mice die in utero due to the same defective vascular development seen in AM- $-1-$ mice, and demonstrated that RAMP2 is the key determinant of the vascular functionality of AM [5].

In the present study, we investigated the pathophysiological significance of RAMP2 in cerebral ischemia. To do so, we generated acute and chronic cerebral ischemia models using heterozygous RAMP2 knockout (RAMP2+/-) and wild-type (WT) mice, and evaluated the efficacy of RAMP2 as a therapeutic target in cerebrovascular disease.

\section{Material and methods}

\section{Experimental animals}

RAMP2+/- mice were generated by our group [5]. Because RAMP2- / - mice die in utero, we used male RAMP2+/- and their WT littermates (10 to 12-week-old male mice for MCAO and 9-week-old male mice for BCAS) in this study. All animal handling procedures were performed in accordance with a protocol approved by the Ethics Committee of Shinshu University.

\section{Middle cerebral artery occlusion (MCAO)}

MCAO, an operative procedure to cause acute cerebral ischemia, was performed in WT and RAMP2+/- mice as described by Shah
A

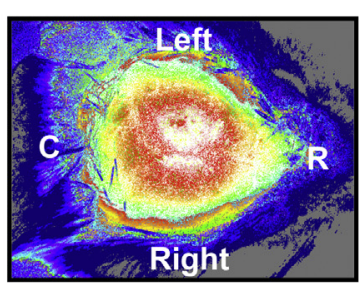

B

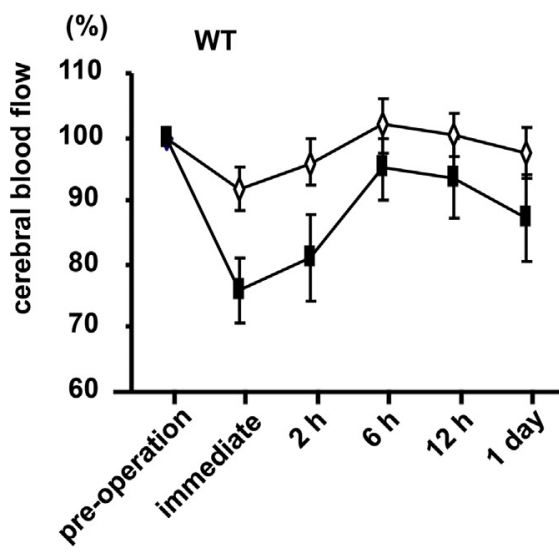

RAMP2 +/-

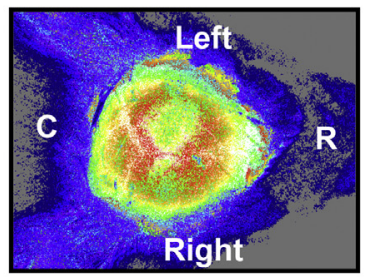

(\%) RAMP2 +/-

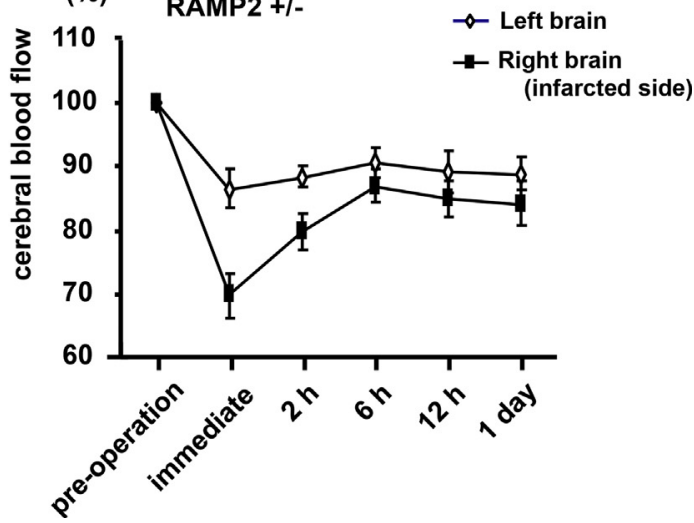

Fig. 1. Evaluation of blood flow recovery after MCAO. (A) Representative laser Doppler images of cerebral blood flow (CBF) in wild-type (WT) and RAMP2+/- mice $24 \mathrm{~h}$ after MCAO. R and C = rostral and caudal side, respectively. (B) Temporal profile of CBF after MCAO in WT and RAMP2+/- mice. CBF in each hemisphere prior to the operation was assigned a value of $100 \%$. Values are expressed as the mean \pm SEM. $n=11$ in each. CBF recovery tended to be delayed in RAMP2 $+/-$ mice on both the infarcted and contralateral sides. 


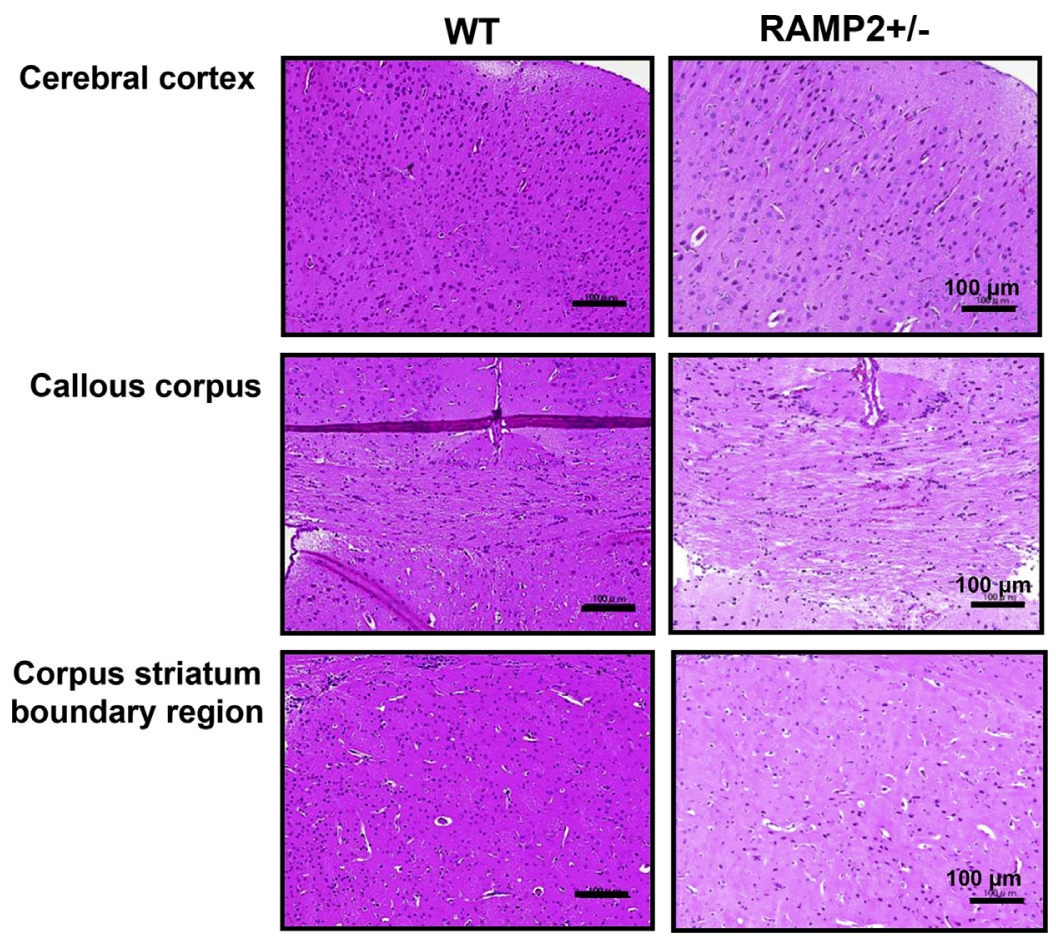

Fig. 2. Histological changes after MCAO. H\&E staining of the cerebral cortex, callous corpus and corpus striatum boundary region in WT and RAMP2+/- mice $24 \mathrm{~h}$ after MCAO. Scale bar $=100 \mu \mathrm{m}$. On the whole, staining was fainter in RAMP2 $+/-$ than WT mice.



B
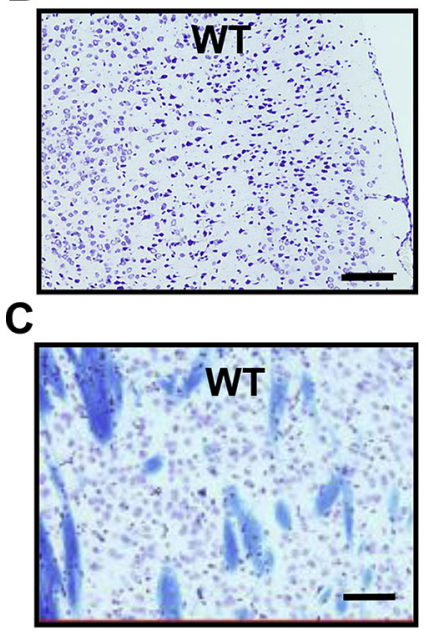
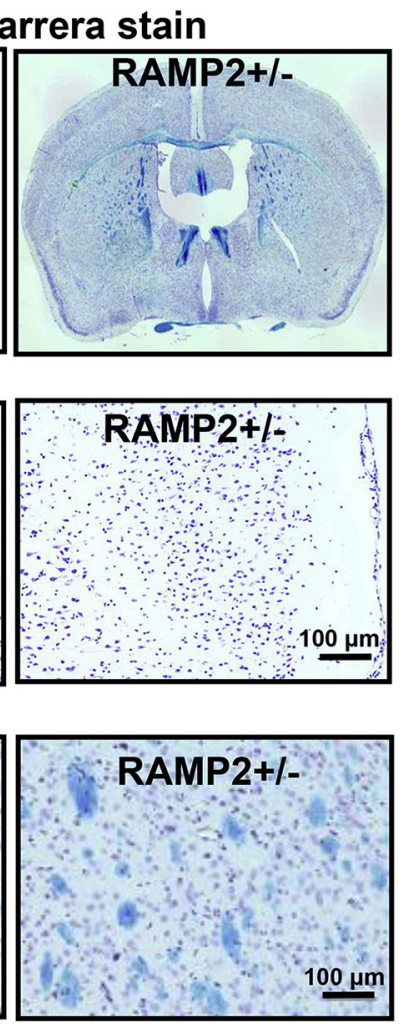

D

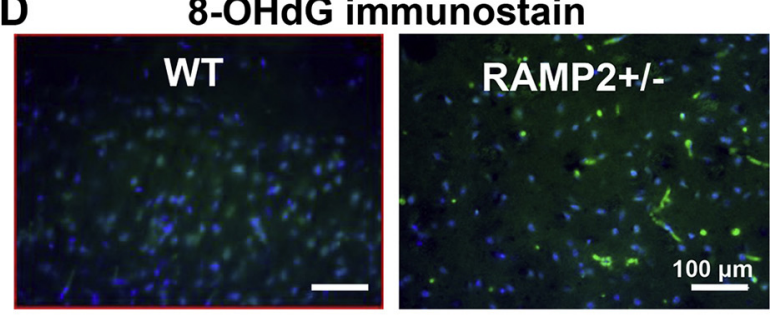

E

TUNEL
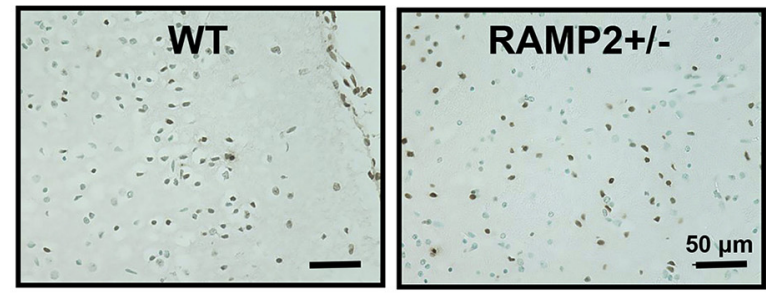

$\mathbf{F}$
TUNEL-positive cells/field

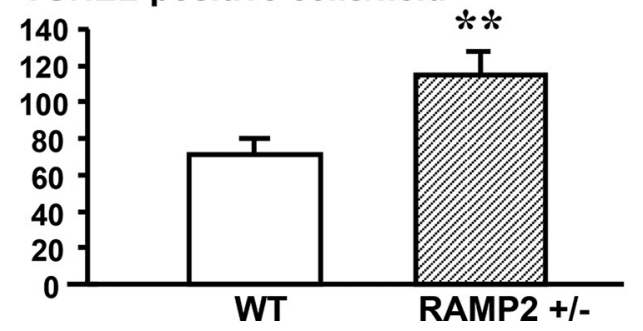

Fig. 3. Evaluation of neuronal cell degeneration and oxidative stress after MCAO. (A-C) Kluver-Barrera staining in coronal plane sections of whole brain (A), cerebral cortex (B), and corpus striatum boundary region (C) after MCAO. Scale bar $=100 \mu \mathrm{m}$. Numbers of cresyl violet-stained neurons at cerebral cortex (B) and luxol fast blue-stained myelin at corpus striatum boundary region (B) were decreased in RAMP2+/- mice. (D) Immunostaining of 8-OHdG, a marker of oxidative DNA damage (shown in green fluorescent). Scale bar $=100 \mu \mathrm{m}$. (E) TUNEL in sections of cerebral cortex. Cells were counter-stained with methyl green. Scale bar $=50 \mu \mathrm{m}$. (F) Bar graph comparing numbers of TUNEL-positive cells per microscopic field (magnification: $200 \times$ ) between WT and RAMP $2+1-$ mice. Bars are mean \pm SEM. $n=6$ in each mouse. $p<0.01$. The incidence of TUNEL positivity was significantly higher in RAMP2+/- than WT mice. 

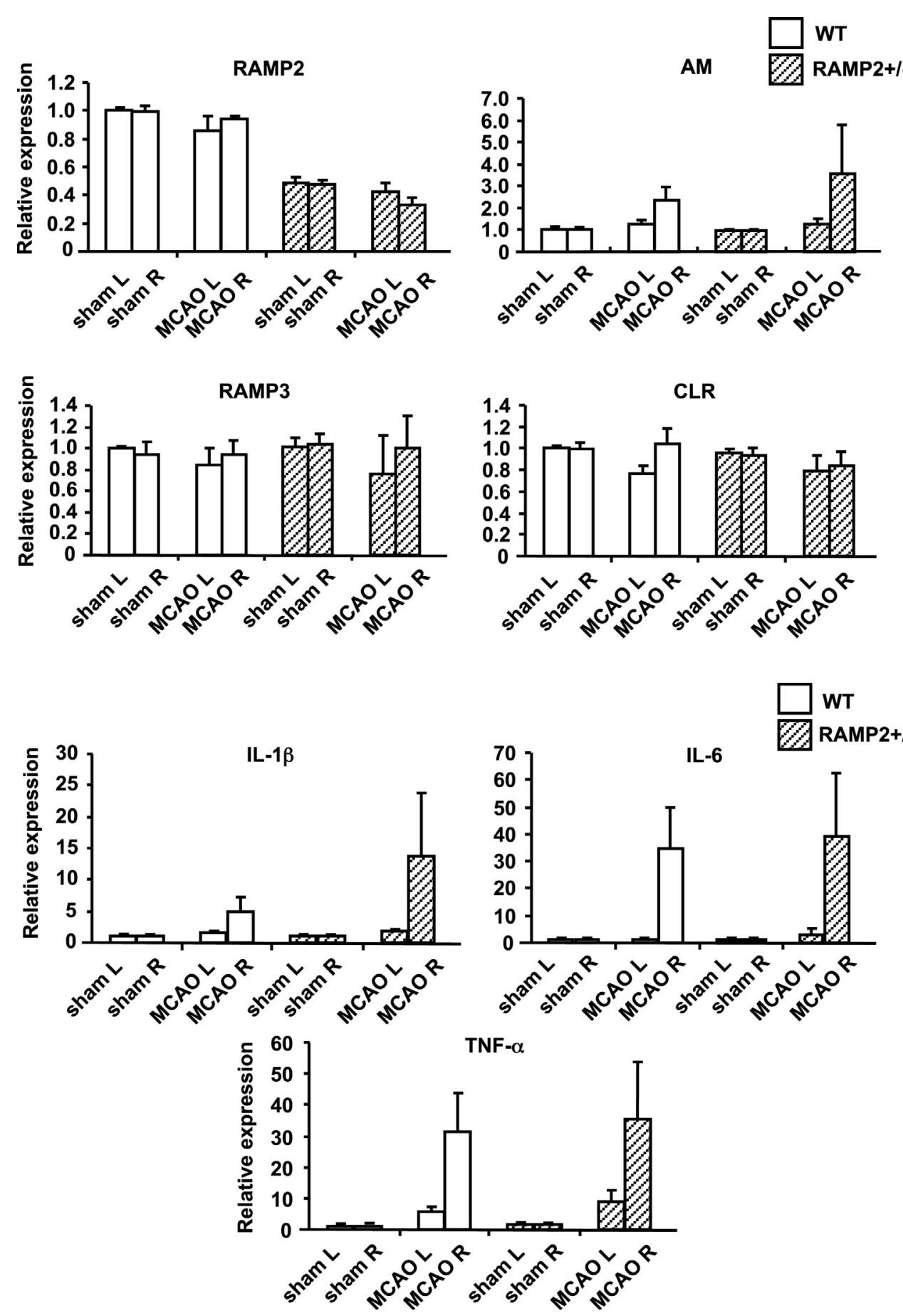

Fig. 4. Evaluation of gene expression after MCAO. Quantitative real-time PCR analysis of the cerebral expression of the indicated genes in WT or RAMP2+/- mice after MCAO or sham operation. Data from the non-infarcted cerebral hemisphere of sham-operated WT mice were assigned a value of 1 . $L$ and $R=$ left and right hemisphere, respectively. Bars are mean \pm SEM. $n=4$ in each.

et al. [28]. Briefly, 1.5-cm of 6-0 nylon surgical suture was coated with silicone to make an embolic filament. Mice were anesthetized with isoflurane, and the right common carotid artery was exposed. Blood flow in the external carotid artery was permanently intercepted using an electric knife. The common carotid artery was clipped, intercepting the blood flow, and the embolic filament was inserted from the external carotid artery into the middle cerebral artery. The filament was then removed after $2 \mathrm{~h}$.

Cerebral blood flow (CBF) was measured using an OMEGAZONE OZ-1 laser Doppler blood flow imaging system (Omegawave Inc.,
Japan). After $24 \mathrm{~h}$, the brains were removed for pathology and gene expression analysis.

Bilateral common carotid artery stenosis (BCAS) and behavioral analysis

BCAS is an operative procedure to cause chronic cerebral ischemia by attaching an external microcoil (internal diameter: $0.18 \mathrm{~mm}$ ) to the bilateral common carotid arteries. We performed BCAS in WT and RAMP2+/- mice as described previously [13,29]. Briefly, mice were anesthetized with isoflurane, and the common 

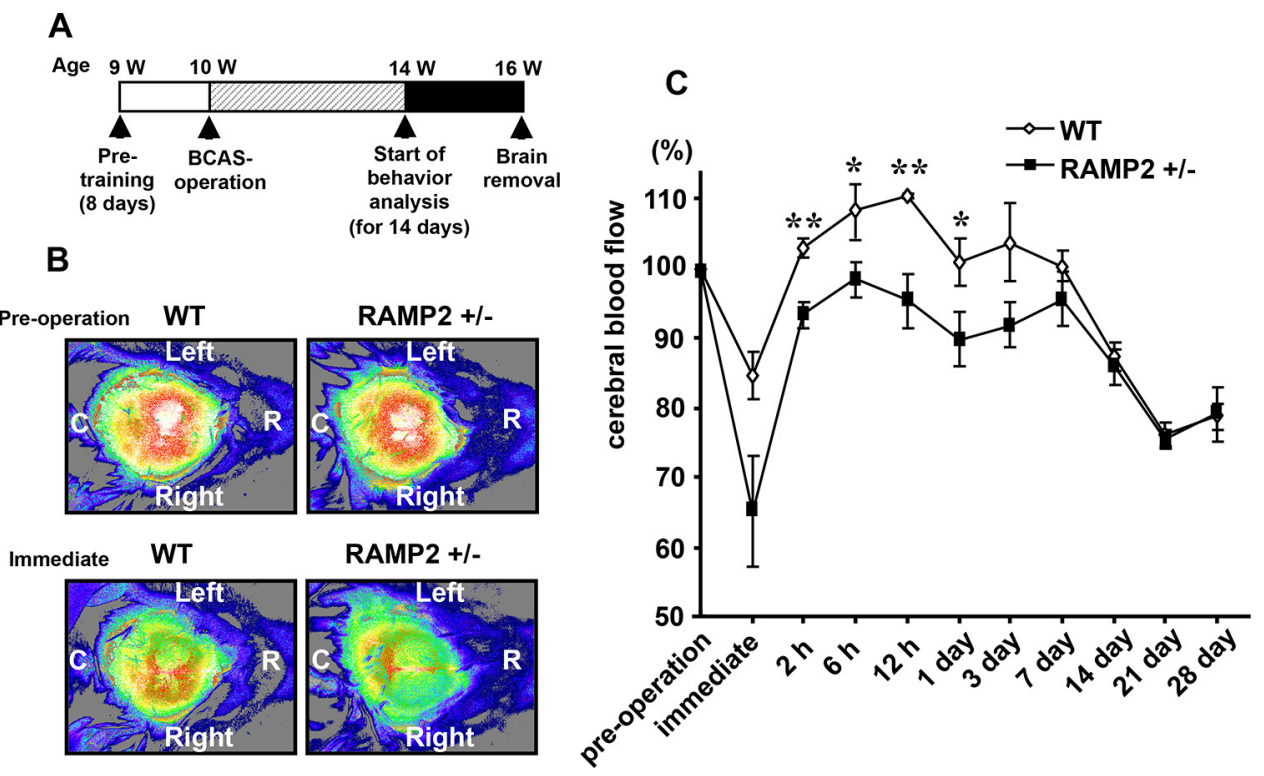

Fig. 5. Evaluation of blood flow recovery during BCAS. (A) Protocol for the evaluation of chronic cerebral ischemia. (B) Representative laser Doppler images of CBF in WT and RAMP2+/- brains before (upper panels) and immediately after (lower panels) induction of BCAS. R and C= rostral and caudal side, respectively. (C) Temporal profile of CBF after BCAS in WT and RAMP2+/-. CBF in each hemisphere prior to the operation was assigned a value of $100 \%$. Values are expressed as mean \pm SEM. ${ }^{* *} p<0.01,{ }^{*} p<0.05 . n=5$ in each. Both mice showed acute reductions of CBF immediately after the operation, which then gradually recovered. By the end of the study (day 28 ), CBF reached about $80 \%$ of the pre-operative level. RAMP2 $+/-$ mice showed a greater reduction of CBF at an early stage after the operation (2-24 $\mathrm{h})$.

carotid artery was exposed. Two 4-0 silk sutures were then placed loosely around distal and proximal parts of the artery and, using the sutures, the artery was lifted, enabling the microcoil to be attached below the carotid bifurcation. Sham animals were subjected to the same surgery, but the microcoil was not attached. CBF was measured using a laser Doppler blood flow imaging system prior to the operation; immediately after the operation; 2,6 , and $12 \mathrm{~h}$ after the operation; and 1, 3, 7, 14, 21 and 28 days after the operation.

Beginning 28 days after attaching the microcoil, we evaluated the behavior of each mouse using an 8-arm radial maze (SMART v3.0.00, Panlab, Spain). For the task, the mice were pretrained to find food pellets within the maze. In the behavioral trials, we counted the numbers of correct choices (visits to an arm with a food pellet) during the initial 4 choices in the maze. We also recorded reference memory errors (counts of visits to arms without a food pellet) and the average time required to take all the food pellets. After the behavioral trials, the brains were removed for pathology and gene expression analysis.

\section{Histology}

Tissues were fixed overnight in $4 \%$ paraformaldehyde, embedded in paraffin, and cut into $7-\mu \mathrm{m}$-thick sections for histological examination. The specimens were then deparaffinized for hematoxylin/eosin (H\&E) staining, cresyl violet staining, Kluver-Barrera staining, and TUNEL. Immunostaining was performed using antibodies against glial fibrillary acidic protein (GFAP; astrocyte marker, Invitrogen), 8-hydroxy-2'-deoxyguanosine (8-OHdG; DNA oxidation breakdown marker, Japan Institute for the Control of Aging), and anti-CD31 (capillary marker, BD Biosciences).

\section{RNA extraction and quantitative real-time RT-PCR}

Total RNA was extracted from tissues using TRI Reagent (Molecular Research Center, Inc.), after which the RNA was treated with DNA-free (Ambion) to remove contaminating DNA and reverse transcribed using a High Capacity cDNA Reverse Transcription Kit (Applied Biosystems). Quantitative real-time RT-PCR was carried out using an Applied Biosystems 7300 real-time PCR System with SYBR green (Toyobo, Japan) or Realtime PCR Master Mix (Toyobo) and TaqMan probes (MBL). The primers and probes used are listed in Table 1. Values were normalized to mouse GAPDH (PreDeveloped TaqMan assay reagents, Applied Biosystems).

\section{Statistical analysis}

Values are expressed as the mean \pm SEM. Student's $t$-test was used to determine the significance of differences between two means. Values of $p<0.05$ were considered significant.

\section{Results}

Blood flow recovery after MCAO was delayed in RAMP2+/- mice

We used an MCAO model to evaluate the effect of acute cerebral infarction in WT and RAMP2+/- mice. When we compared cerebral blood flow (CBF) $24 \mathrm{~h}$ after MCAO, the blood flow recovery tended to be lower in RAMP2 $+/-$ mice (Fig. $1 \mathrm{~A}$ ). Immediately after the operation, both the infarcted and contralateral sides of the brain showed transiently diminished blood flow. In WT mice, blood flow then rapidly recovered on the contralateral side. In contrast, in RAMP2+1- mice, blood flow recovery on the contralateral side appeared to be greatly affected by the poor recovery on the infarcted side (Fig. 1B) - i.e., blood flow to both the infarcted side and contralateral side remained diminished $24 \mathrm{~h}$ after the MCAO operation.

Greater neural cell damage and oxidative stress in RAMP2+/mice after MCAO operation

Histological analysis $24 \mathrm{~h}$ after MCAO revealed reductions in cell density, cellular vacuolation and nuclear alterations in the cerebral cortex, callous corpus and corpus striatum boundary region in both RAMP+/- and WT mice, but the damage was more severe in RAMP2+/- mice (Fig. 2). In Kluver-Barrera staining of the brain sections (Fig. 3A-C), the number of cresyl violet-stained neurons 



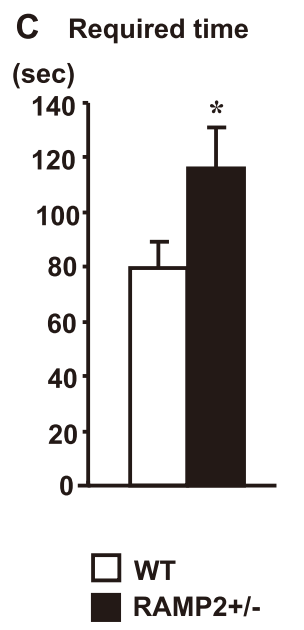

D

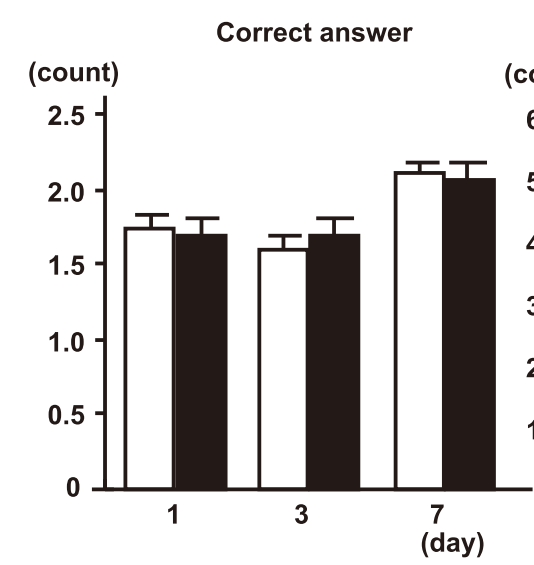

E

Reference memory error

\section{$\mathbf{F}$}

(sec)

Required time

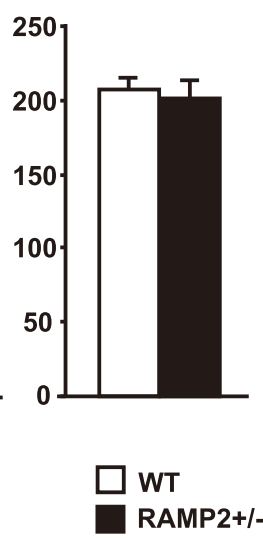

Fig. 6. Behavioral analysis after BCAS. (A-C) Eight-arm radial maze tests were performed after 28 days of BCAS. Behavior trials were performed on the indicated days after measuring CBF on day 28. (A) Comparison of correct answer counts during the initial 4 choices. (B) Comparison of reference memory error; counts of visits to an arm without a food pellet. The errors were counted for $5 \mathrm{~min}$ or until all the pellets were eaten. (C) Comparison of the average time required to take all of the food pellets during the 2 weeks of the behavior trials. Bars are mean \pm SEM. WT: $n=13$; RAMP2 $+1-: n=11$ in A and B. WT: $n=9$, RAMP $+1-: n=7$ in C. The test was performed 2 times a day and the mean value was used for the statistical analysis. ${ }^{*} p<0.05$. (D-F) Results of 8 -arm radial maze tests during pre-training period; correct answer (D), reference memory error (E), and required time (F). Bars are mean \pm SEM. WT: $n=42$, RAMP2 $+1-: n=32$.

at the cerebral cortex (Fig. 3B) and luxol fast blue-stained myelin at the corpus striatum boundary region (Fig. 3C) were decreased in RAMP2+/ - mice. Oxidative stress was assessed on the infarcted side by immunostaining for 8-OHdG, levels of which are indicative of the degree of oxidative DNA damage. We found that 8-OHdG levels were higher in RAMP2+/- than WT brains (Fig. 3D). Furthermore, TUNEL analysis of the infarcted area showed a significantly higher incidence of apoptosis in RAMP2+/- brains than in WT brains (Fig. 3E and F), indicating greater irreversible brain damage. Taken together, these findings indicate RAMP2+/- mice are more vulnerable to acute brain ischemia than WT mice.

\section{Altered gene expression in RAMP2+/- mice following MCAO}

We next evaluated the gene expression in the infarcted and contralateral cerebral hemispheres after MCAO or sham operation (Fig. 4). In RAMP2+/- mice, RAMP2 gene expression was reduced to about half of that seen in WT mice. On the other hand, CLR and another RAMP isoform, RAMP3, were unchanged. AM expression was increased on the infarcted side in both genotypes, but the elevation was pronounced in RAMP2 $+/-$ mice, which suggests a compensatory response to the reduction of AM-RAMP2 signaling in RAMP2+/- mice.

Although the difference was not statistically significant, the gene expression level of IL-1 $\beta$ was slightly higher in RAMP2+/following MCAO. Thus, RAMP2+1- mice showed more severe brain damage with greater inflammation after acute ischemia than WT mice.

Blood flow recovery after BCAS was delayed in RAMP2+/- mice

Fig. 5A shows the protocol used for the evaluation of chronic cerebral ischemia using a BCAS model. Nine-week-old WT and RAMP2+/- mice underwent pre-training in an 8-arm radial maze for 8 days. The BCAS operation was then performed and blood flow recovery was followed for 28 days. At 14 weeks, behavior was analyzed using the 8 -arm radial maze and at 16 weeks the brains were removed for further analysis. Immediately after the BCAS operation, CBF was depressed in both WT and RAMP2+/- mice (Fig. 5B and C). During the period spanning $2 \mathrm{~h}$ to $12 \mathrm{~h}$ after the operation, the 
A


C

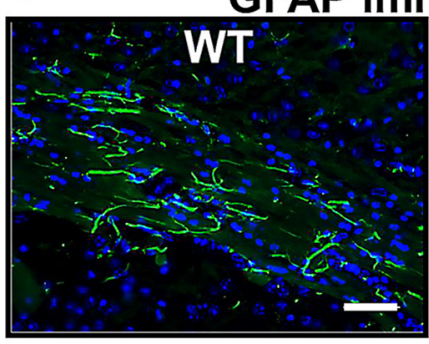

GFAP immunostain

E

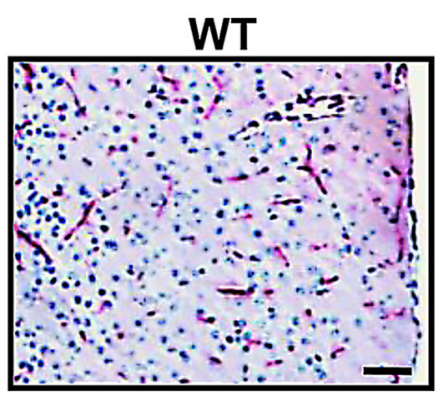



CD31 immunostain

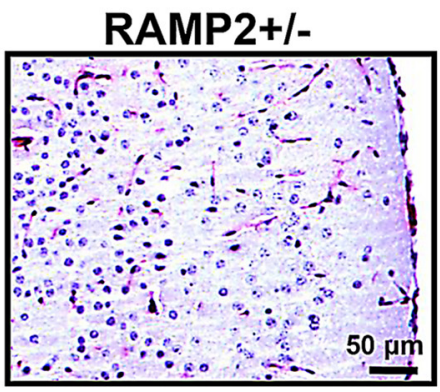

B

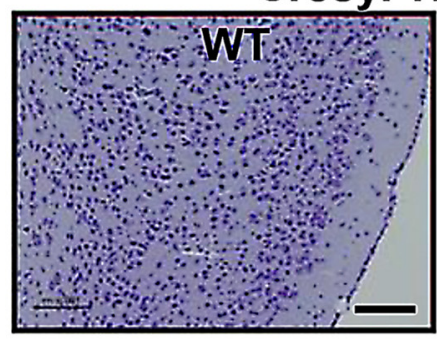

Cresyl violet stain

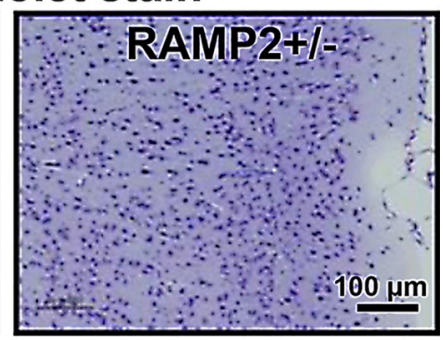

D

8OH-dG immunostain

Cerebral cortex

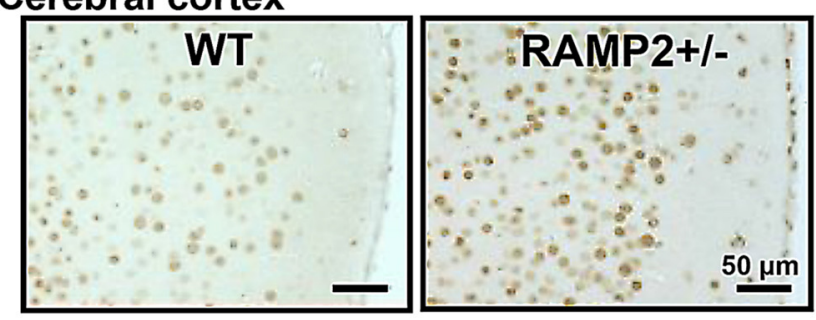

Callous corpus



Corpus striatum boundary region

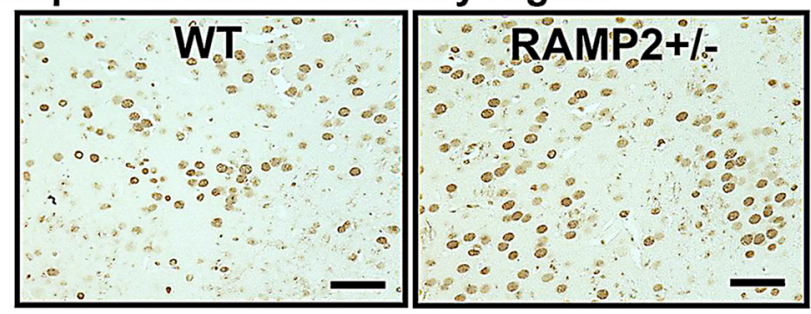

Fig. 7. Histological changes after 42 days of BCAS. (A and B) H\&E (A) and cresyl violet (B) staining of sections of cerebral cortex collected from WT and RAMP2+/- mice after 42 days of BCAS. Scale bar in (A) and (B) =50 $\mu \mathrm{m}$ and $100 \mu \mathrm{m}$, respectively. (C-E) Immunostaining of GFAP-positive astrocytes (C); 8OH-dG, a marker of oxidative DNA damage (D), and CD31-positive capillaries (E). Sections of cerebral cortex (C and E) or cerebral cortex, callous corpus, and corpus striatum boundary region (D) are shown. Green fluorescent in C, brown stain in D, and red stain in E represent positive immunoreactivity. Cell nuclei were stained with DAPI and hematoxylin in (C) and (E), respectively. Scale bar $=50 \mu \mathrm{m}$. RAMP2 $+/$ - brains showed glial cell activation, higher levels of oxidative stress, and retarded capillary formation.

CBF returned to the basal level; however, it then gradually declined until the end of the observation period (day 28). The time-course of the changes in CBF was similar between WT and RAMP2+/- mice, but the flow at an early stage after the BCAS operation (2-24h) was significantly lower in RAMP2+/- mice (Fig. 5C).

Behavior analysis reveals more severe cognitive disorder in RAMP2+/- mice

After monitoring CBF for 28 days, we performed a behavioral analysis using an 8-arm radial maze. The visits to the arm with a food pellet were considered correct, and the number of visits out of the first four choices were counted. Thus the maximum score was 4 (Fig. 6A). At a relatively earlier stage in the behavioral analysis (day 3 ), the counts of correct answers were significantly lower in RAMP2+/- mice than in WT mice. Correspondingly, the counts of visits to an arm without a food pellet (reference memory errors) were significantly higher in RAMP2+/- mice on days 3 and 7 , indicating more severe disturbance of reference memory (Fig. 6B). In addition, the average time required to obtain the food pellets was significantly longer for RAMP2+/- mice (Fig. 6C). Such differences were not detected during the pre-training period (Fig. 6D-F), suggesting lower RAMP2 levels exacerbate the cognitive disorder related to chronic cerebral ischemia.

Greater neuronal cell loss, glial cell activation, oxidative stress, and retarded capillary formation in $\mathrm{RAMP} 2+$ -

Pathological analysis performed after 42 days of BCAS revealed that RAMP2+/- brains showed greater neural cell loss from the 

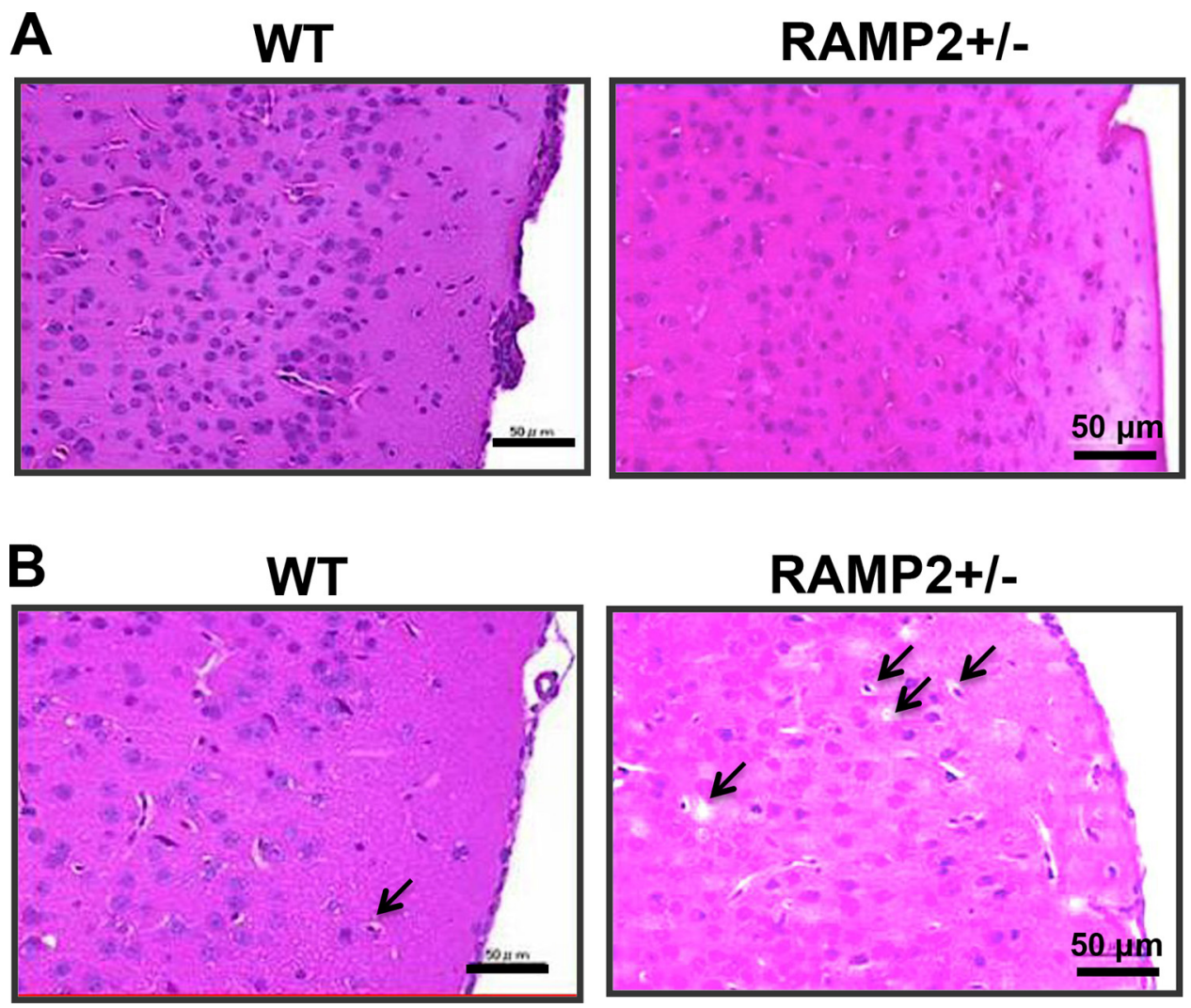

Fig. 8. Histological changes early after BCAS operation. (A and B) H\&E staining in sections of cerebral cortex collected from WT and RAMP2+/- mice 1 day (A) or 5 days (B) after the BCAS operation. Scale bar $=50 \mu \mathrm{m}$. On day 1 , RAMP2 $+/$ - brains showed poorer staining than WT. On day 5 , vacuolar degeneration of neuronal cells (arrows in B) was detected, and was pronounced in RAMP2+/- brains.

cerebral cortex than WT mice (Fig. 7A and B). Likewise, neural cell loss in the callous corpus and corpus striatum boundary region was also greater in RAMP2+/- mice than in WT mice (data not shown). On the other hand, GFAP-positive astrocytes in the cerebral cortex were increased in RAMP2+/- mice (Fig. 7C), indicating glial cell activation. Oxidative stress, indicated by oxidative DNA damage (8-OHdG levels; Fig. 7D) was higher in the cerebral cortex, callous corpus, and corpus striatum boundary region in RAMP2+/-. On the other hand, CD31-positive capillary formation was retarded in the cerebral cortex of RAMP2+/(Fig. 7E).

\section{Histological changes detected early after BCAS in RAMP2+/- mice}

Because there was a large transient fall in CBF early after the BCAS operation, even though the cerebral vessels were not completely blocked, we speculated that histological changes might have occurred earlier than day 42. Consistent with that idea, even on day 1 after the BCAS operation, cell densities were lower in RAMP2+/than WT brains (Fig. 8A). Moreover, on day 5 after the operation, cellular vacuolization was greater in RAMP2+/- than WT brains (Fig. 8B).

\section{Altered gene expression in RAMP2+/- mice subjected to BCAS}

In RAMP2+1- mice, the gene expression of the inflammatory cytokines was slightly higher than WT early after BCAS. This suggests that, as with MCAO, inflammatory responses occurred early after induction of BCAS and were more severe in RAMP2+/- (Fig. 9).

\section{Discussion}

Significant advances have been made in our understanding of the cellular and molecular events underlying ischemic cell death after cerebral infarction, including loss of metabolic stores, excessive intracellular calcium accumulation, oxidative stress, and potentiation of the inflammatory response [40]. We anticipate that knowledge of the underlying cellular and molecular mechanisms will enable development of novel therapeutic approaches to the treatment of cerebral ischemic disease. Within this context, endogenous vasoactive molecules, which work to maintain vascular and organ homeostasis, appear to be attractive therapeutic targets. One of these vasoactive molecules is AM, which, in addition to inducing vasodilation, exerts antiapoptotic, antiinflammatory, antioxidative stress, and organ-protective effects. Indeed, the potential for AM use in the treatment of cerebrovascular diseases has attracted much attention. For instance, using a transient focal ischemia model, Miyamoto et al. found that infarct volumes and neurological deficits were greater in $\mathrm{AM}+\mathrm{C}-$ mice than in WT mice [16]. They also reported that reduced cAMP-protein kinase A signaling in $\mathrm{AM}+/$ - mice leads to increases in oxidative stress, which likely worsens the ischemic brain damage. In addition, Fernandez et al. reported that CNS-specific AM-/- mice were less resistant to hypobaric hypoxia than WT mice, suggesting AM exerts a neuroprotective effect in the CNS [4]. Conversely, using mice overexpressing circulating AM, Maki et al. showed that elevation of AM levels after cerebral infarction accelerated capillary formation, hastened blood flow recovery, and prevented memory deficits [14].

These earlier observations provide compelling evidence that $\mathrm{AM}$ works to prevent cerebral injuries, but they provide no 

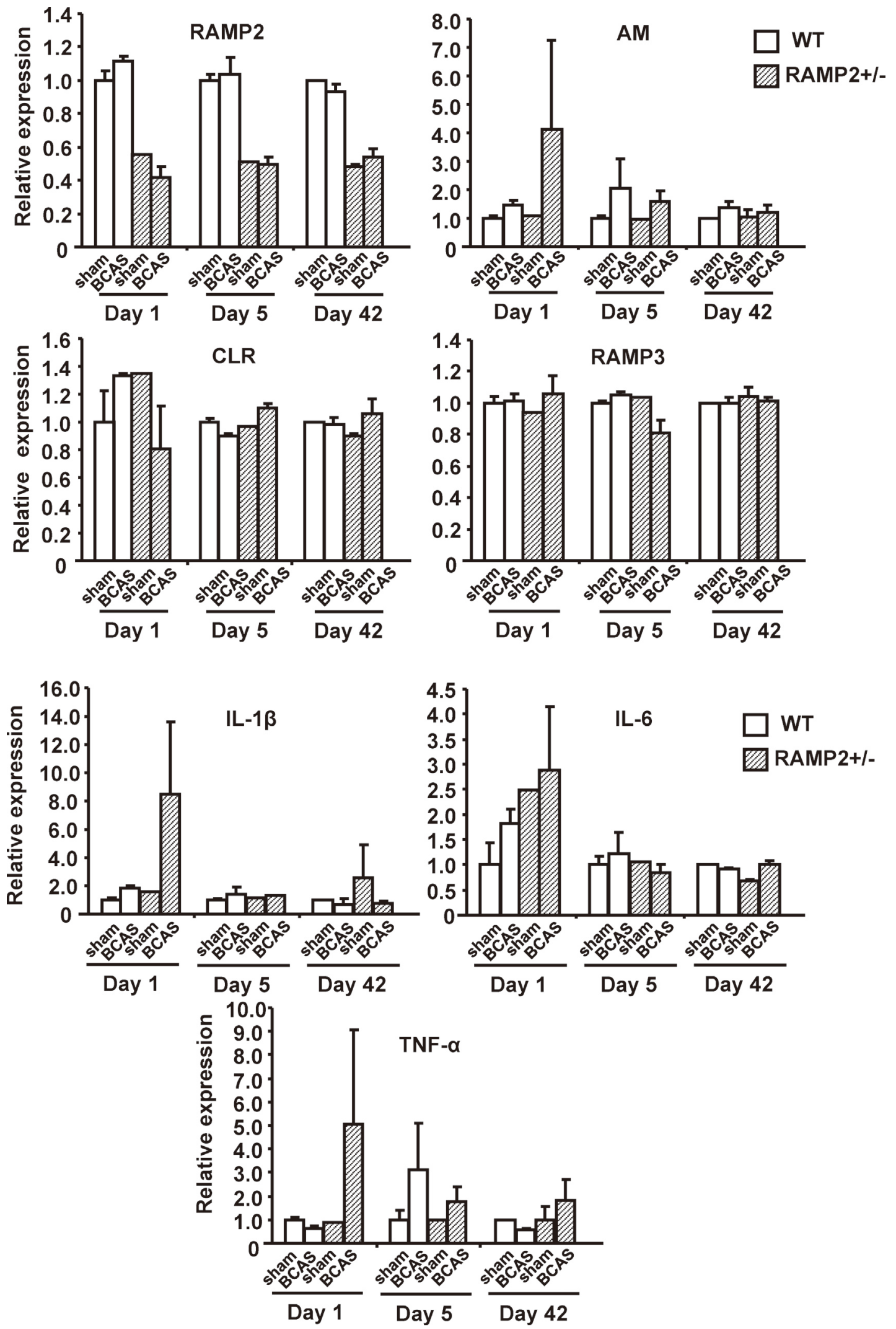

Fig. 9. Evaluation of gene expression after BCAS. Quantitative real-time PCR analysis of expression of the indicated genes in whole brains from WT and RAMP2+/- mice after BCAS or sham operation. Data from the sham-operated WT mice on each day were assigned a value of 1 . Bars are mean \pm SEM. $n=2-4$ in each. 
information about which of the two dimeric receptors of AM, CLR/RAMP2 and/or CLR/RAMP3, mediate those beneficial effects. However, we previously reported that AM- - - and RAMP2 - / - mice die in utero due the same defect in vascular development [5]. In contrast, RAMP1 - / - and RAMP3-/- mice are born normally [1,35]. Moreover, aged RAMP2+/- mice spontaneously develop severe organ fibrosis with accelerated vascular senescence [12]. We therefore postulated that RAMP2 is the key determinant of the vascular functionality of AM. In the present study, we showed that AM acting via CLR/RAMP2 works to prevent brain injury in both acute and chronic cerebral ischemia.

Adrenomedullin was originally isolated as a potent vasodilator, and we reported that AM plays a key role in the physiological regulation of vascular tone $[19,20]$. AM expression was upregulated by hypoxia in an acute cerebral infarction model [27], while intravenous infusion of $A M$ increased $C B F$ and prevented ischemic brain injury [3]. Thus, AM is thought to be upregulated in response to cerebral ischemia as a compensatory response to restore CBF. In the present study, we found that AM gene expression was elevated on the infarcted side after MCAO in both WT and RAMP2+/mice, but the response was more pronounced in RAMP2 $+1-$ mice. This suggests there was positive feedback to offset the reduction of RAMP2, and thus AM-CLR/RAMP2 signaling, and that RAMP2 plays a central role with AM after cerebral infarction. In addition, $\mathrm{CBF}$ was also depressed on the contralateral side after cerebral infarction in RAMP2+/- mice. This likely reflects the fact that, even without vascular occlusion, anesthesia and operative procedures often affect $\mathrm{CBF}$, and again RAMP2+/- mice were more sensitive to these procedures. Taken together, these results indicate that the endogenous AM-RAMP2 system plays a protective role to maintain CBF after acute cerebral infarction.

Inflammatory cytokines are important factors contributing to ischemic brain injury [2]. AM has been shown to suppress inflammation [8]. In the present study, we found that the gene expression of inflammatory cytokines was slightly higher in RAMP2+/- mice after MCAO and early after BCAS. Inflammation has been implicated as a secondary mechanism underlying ischemia-induced neuronal injury, extending the area of brain infarcts $[38,39]$. Consistent with those reports, we observed that activation of astrocytes in the BCAS model was enhanced in RAMP2+/- mice, likely exacerbating inflammation and neural cell injury [33,36]. With loss of RAMP2, therefore, not only do the diminished vascular effects contribute to the severity of brain damage, but so does the enhanced inflammatory response.

Inflammation and oxidative stress are closely related in cerebrovascular disease, acting together to exacerbate its pathology [15]. Shimosawa et al. first reported the antioxidative function of AM through the suppression of NADPH oxidase [30,31]. We reported that aged RAMP2 $+/-$ mice showed severe organ fibrosis with marked oxidative stress and accelerated vascular senescence. Conversely, endothelial cells stably overexpressing RAMP2 were protected from cellular senescence induced by oxidative stress [12]. In the present study, we found that the level of oxidative stress marker was higher in RAMP2+/- mice than in WT mice, suggesting the antioxidative effects of the endogenous AM-RAMP2 system are also protective against ischemic brain damage. Collectively then, our findings indicate that the AM-RAMP2 system protects against brain injury by restoring $\mathrm{CBF}$ and suppressing both inflammation and oxidative stress.

There are currently no effective treatments for vascular dementia, which is an aftereffect of cerebral infarction - i.e., there are as yet no treatments that can restore cerebral perfusion or the functionality of cerebral vessels. Consequently, enhancement of angiogenesis could be the most promising approach to treating vascular dementia. In BCAS-induced chronic cerebral ischemia, we found that RAMP2+/- mice showed reduced capillary development. We also found that reducing RAMP2 worsened the cognitive disorder associated with chronic cerebral ischemia. Maki et al. reported that AM promotes angiogenesis and prevents cognitive decline after chronic cerebral hypoperfusion in mice [13]. We also reported that $\mathrm{AM}+1-$ mice showed reduced blood flow and capillary formation in tumor transplantation and hind-limb ischemia models [6]. AM possesses novel angiogenic properties mediated by its ability to enhance VEGF expression [7]. However, during the chronic phase after BCAS, we detected no change in VEGF expression (data not shown). We believe that the angiogenic potency of AM does not solely reflect its regulation of angiogenic factors; we reported that RAMP2-/- mice also showed disruption of cellular adhesion, tight junctions, and the actin cytoskeleton of vascular endothelial cells $[5,12]$.

AM has been attracting much attention for its potential clinical application. On the other hand, the clinical applicability of AM, like that of other endogenous bioactive peptides, has limitations; AM has a very short half-life in the blood, which makes it impractical for use in the treatment of chronic diseases, such as vascular dementia. In the present study, we showed that through maintenance of CBF and suppression of inflammation and oxidative stress, endogenous RAMP2, a component of the dimeric AM receptor (CLR/RAMP2), works to prevent tissue damage after cerebral ischemia, thereby contributing to the maintenance of cognitive function. We therefore suggest that the CLR/RAMP2 complex or RAMP2 itself could be a useful therapeutic target for the prevention or treatment of cerebrovascular diseases, including both cerebral infarction and vascular dementia. It is noteworthy that RAMP2 is the critical determinant of the protective effects of AM in cerebral ischemia. In that context, our findings provide a clear rationale for the development of drugs that modulate RAMP2 and thus the vascular functionality of AM.

\section{Acknowledgements}

This study was supported by the Funding Program for Next Generation World-Leading Researchers (NEXT Program) grant No. (LS053) from the Cabinet Office, Government of (26293183, 26293085, 25460330, 26460337, 26670153) Cardiovascular Diseases from the National Cardiovascular Center, and research grants from the Novartis Foundation for Gerontological Research, the Ichiro Kanehara Foundation, the Cosmetology Research Foundation, the SENSHIN Medical Research Foundation, the Nagao Memorial Fund, the Kanzawa Medical Research Foundation, the Ono Medical Research Foundation, the Nakatomi Foundation, the Japan Heart Foundation \& Astellas/Pfizer Grant for Research on Atherosclerosis Update, the Research Foundation for Opto-Science and Technology, Takeda Science Foundation, and the Japan Vascular Disease Research Foundation.

\section{References}

[1] Dackor R, Fritz-Six K, Smithies O, Caron K. Receptor activity-modifying proteins 2 and 3 have distinct physiological functions from embryogenesis to old age. J Biol Chem 2007;282:18094-9.

[2] del Zoppo G, Ginis I, Hallenbeck JM, Iadecola C, Wang X, Feuerstein GZ. Inflammation and stroke: putative role for cytokines, adhesion molecules and iNOS in brain response to ischemia. Brain Pathol 2000;10:95-112.

[3] Dogan A, Suzuki Y, Koketsu N, Osuka K, Saito K, Takayasu M, et al. Intravenous infusion of adrenomedullin and increase in regional cerebral blood flow and prevention of ischemic brain injury after middle cerebral artery occlusion in rats. J Cereb Blood Flow Metab 1997;17:19-25.

[4] Fernandez AP, Serrano J, Tessarollo L, Cuttitta F, Martinez A. Lack of adrenomedullin in the mouse brain results in behavioral changes, anxiety, and lower survival under stress conditions. Proc Natl Acad Sci U S A 2008; $105: 12581-6$

[5] Ichikawa-Shindo Y, Sakurai T, Kamiyoshi A, Kawate H, Iinuma N, Yoshizawa T, et al. The GPCR modulator protein RAMP2 is essential for angiogenesis and vascular integrity. J Clin Invest 2008;118:29-39. 
[6] Iimuro S, Shindo T, Moriyama N, Amaki T, Niu P, Takeda N, et al. Angiogenic effects of adrenomedullin in ischemia and tumor growth. Circ Res 2004;95:415-23.

[7] Iinuma N, Sakurai T, Kamiyoshi A, Ichikawa-Shindo Y, Arai T, Yoshizawa T, et al Adrenomedullin in sinusoidal endothelial cells play protective roles against cold injury of liver. Peptides 2010;31:865-71.

[8] Isumi Y, Kubo A, Katafuchi T, Kangawa K, Minamino N. Adrenomedullin suppresses interleukin-1beta-induced tumor necrosis factor-alpha production in Swiss 3T3 cells. FEBS Lett 1999;463:110-4.

[9] Iwasaki H, Eguchi S, Shichiri M, Marumo F, Hirata Y. Adrenomedullin as a novel growth-promoting factor for cultured vascular smooth muscle cells: role of tyrosine kinase-mediated mitogen-activated protein kinase activation. Endocrinology 1998;139:3432-41.

[10] Kano H, Kohno M, Yasunari K, Yokokawa K, Horio T, Ikeda M, et al. Adrenomedullin as a novel antiproliferative factor of vascular smooth muscle cells. J Hypertens 1996;14:209-13.

[11] Kitamura K, Kangawa K, Kawamoto M, Ichiki Y, Nakamura S, Matsuo $\mathrm{H}$, et al. Adrenomedullin: a novel hypotensive peptide isolated from human pheochromocytoma. Biochem Biophys Res Commun 1993;192:55360.

[12] Koyama T, Ochoa-Callejero L, Sakurai T, Kamiyoshi A, Ichikawa-Shindo Y, Iinuma N, et al. Vascular endothelial adrenomedullin-RAMP2 system is essential for vascular integrity and organ homeostasis. Circulation 2013;127: $842-53$.

[13] Maki T, Ihara M, Fujita Y, Nambu T, Harada H, Ito H, et al. Angiogenic roles of adrenomedullin through vascular endothelial growth factor induction. Neuroreport 2011;22:442-7

[14] Maki T, Ihara M, Fujita Y, Nambu T, Miyashita K, Yamada M, et al. Angiogenic and vasoprotective effects of adrenomedullin on prevention of cognitive decline after chronic cerebral hypoperfusion in mice. Stroke 2011;42:1122-8.

[15] Miller AA, Budzyn K, Sobey CG. Vascular dysfunction in cerebrovascular disease: mechanisms and therapeutic intervention. Clin Sci (Lond) 2010:119:1-17.

[16] Miyamoto N, Tanaka R, Shimosawa T, Yatomi Y, Fujita T, Hattori N, et al. Protein kinase A-dependent suppression of reactive oxygen species in transient focal ischemia in adrenomedullin-deficient mice. J Cereb Blood Flow Metab 2009;29:1769-79.

[17] Miyashita K, Itoh H, Sawada N, Fukunaga Y, Sone M, Yamahara K, et al. Adrenomedullin promotes proliferation and migration of cultured endothelial cells. Hypertens Res 2003;26(Suppl.):S93-8.

[18] Murphy TC, Samson WK. The novel vasoactive hormone, adrenomedullin, inhibits water drinking in the rat. Endocrinology 1995;136:2459-63.

[19] Nishimatsu H, Hirata Y, Shindo T, Kurihara H, Kakoki M, Nagata D, et al. Role of endogenous adrenomedullin in the regulation of vascular tone and ischemic renal injury: studies on transgenic/knockout mice of adrenomedullin gene. Circ Res 2002;90:657-63.

[20] Nishimatsu H, Hirata Y, Shindo T, Kurihara H, Suzuki E, Sata M, et al. Endothelial responses of the aorta from adrenomedullin transgenic mice and knockout mice. Hypertens Res 2003;26(Suppl.):S79-84.

[21] Niu P, Shindo T, Iwata H, Ebihara A, Suematsu Y, Zhang Y, et al. Accelerated cardiac hypertrophy and renal damage induced by angiotensin II in adrenomedullin knockout mice. Hypertens Res 2003;26:731-6.
[22] Niu P, Shindo T, Iwata H, Iimuro S, Takeda N, Zhang Y, et al. Protective effects of endogenous adrenomedullin on cardiac hypertrophy, fibrosis, and renal damage. Circulation 2004;109:1789-94.

[23] Okumura H, Nagaya N, Kangawa K. Adrenomedullin infusion during ischemia/reperfusion attenuates left ventricular remodeling and myocardial fibrosis in rats. Hypertens Res 2003;26(Suppl.):S99-104.

[24] Petrie MC, Hillier C, Morton JJ, McMurray JJ. Adrenomedullin selectively inhibits angiotensin II-induced aldosterone secretion in humans. J Hypertens 2000;18:61-4.

[25] Samson WK, Murphy T, Schell DA. A novel vasoactive peptide, adrenomedullin, inhibits pituitary adrenocorticotropin release. Endocrinology 1995; 136:2349-52.

[26] Samson WK, Murphy TC. Adrenomedullin inhibits salt appetite. Endocrinology 1997;138:613-6.

[27] Serrano J, Alonso D, Encinas JM, Lopez JC, Fernandez AP, Castro-Blanco S, et al. Adrenomedullin expression is up-regulated by ischemia-reperfusion in the cerebral cortex of the adult rat. Neuroscience 2002;109:717-31.

[28] Shah ZA, Namiranian K, Klaus J, Kibler K, Dore S. Use of an optimized transient occlusion of the middle cerebral artery protocol for the mouse stroke model. J Stroke Cerebrovasc Dis 2006;15:133-8.

[29] Shibata M, Ohtani R, Ihara M, Tomimoto H. White matter lesions and glial activation in a novel mouse model of chronic cerebral hypoperfusion. Stroke 2004;35:2598-603.

[30] Shimosawa T, Ogihara T, Matsui H, Asano T, Ando K, Fujita T. Deficiency of adrenomedullin induces insulin resistance by increasing oxidative stress. Hypertension 2003;41:1080-5.

[31] Shimosawa T, Shibagaki Y, Ishibashi K, Kitamura K, Kangawa K, Kato S, et al. Adrenomedullin, an endogenous peptide, counteracts cardiovascular damage. Circulation 2002;105:106-11.

[32] Shindo T, Kurihara Y, Nishimatsu H, Moriyama N, Kakoki M, Wang Y, et al. Vascular abnormalities and elevated blood pressure in mice lacking adrenomedullin gene. Circulation 2001;104:1964-71.

[33] Stoll G, Jander S, Schroeter M. Inflammation and glial responses in ischemic brain lesions. Prog Neurobiol 1998;56:149-71.

[34] Taylor GM, Meeran K, O‘Shea D, Smith DM, Ghatei MA, Bloom SR. Adrenomedullin inhibits feeding in the rat by a mechanism involving calcitonin gene-related peptide receptors. Endocrinology 1996;137:3260-4.

[35] Tsujikawa K, Yayama K, Hayashi T, Matsushita H, Yamaguchi T, Shigeno T, et al. Hypertension and dysregulated proinflammatory cytokine production in receptor activity-modifying protein 1-deficient mice. Proc Natl Acad Sci U S A 2007; 104:16702-7.

[36] Tuppo EE, Arias HR. The role of inflammation in Alzheimer's disease. Int J Biochem Cell Biol 2005;37:289-305.

[37] Uzan B, Villemin A, Garel JM, Cressent M. Adrenomedullin is anti-apoptotic in osteoblasts through CGRP1 receptors and MEK-ERK pathway. J Cell Physiol 2008;215:122-8.

[38] Wen YD, Zhang HL, Qin ZH. Inflammatory mechanism in ischemic neuronal injury. Neurosci Bull 2006;22:171-82.

[39] Zaremba J. Contribution of tumor necrosis factor alpha to the pathogenesis of 33 stroke. Folia Morphol (Warsz) 2000;59:137-43.

[40] Zheng Z, Lee JE, Yenari MA. Stroke: molecular mechanisms and potential targets for treatment. Curr Mol Med 2003;3:361-72. 